\title{
Sarcopenia as an independent predictor of the surgical outcomes of patients with inflammatory bowel disease: a meta-analysis
}

\author{
Adrienn Erős ${ }^{1,2} \cdot$ Alexandra Soós $s^{1,3} \cdot$ Péter Hegyi $^{1,3,4,5} \cdot$ Zsolt Szakács $^{1,2} \cdot$ Márton Benke $^{6} \cdot$ Ákos Szűcs ${ }^{6}$. \\ Petra Hartmann ${ }^{7}$. Bálint Erőss ${ }^{1,2} \cdot$ Patricia Sarlós ${ }^{1,2,5}$
}

Received: 31 May 2019 / Accepted: 8 September 2019 / Published online: 15 October 2019

(C) The Author(s) 2019

\begin{abstract}
Patients with inflammatory bowel disease (IBD) are at risk of sarcopenia, which is associated with poor clinical outcomes. We conducted this study to assess whether sarcopenia predicts the need for surgery and postoperative complications in patients with IBD. We performed a systematic search of four electronic databases, last updated in March, 2019. Data from studies comparing rates of surgery and postoperative complications in sarcopenic IBD patients versus non-sarcopenic IBD patients were pooled with the random-effects models. We calculated the odds ratios (OR) with a 95\% confidence interval (CI). Ten studies with a collective total of 885 IBD patients were included in our meta-analysis. Although the analysis of raw data did not reveal significant differences between the two groups with respect to the rate of surgery and postoperative complications $(\mathrm{OR}=1.826 ; 95 \% \mathrm{CI} 0.913-3.654 ; p=0.089$ and $\mathrm{OR}=3.265 ; 95 \% \mathrm{CI} 0.575-18.557 ; p=0.182$, respectively $)$, the analysis of adjusted data identified sarcopenia as an independent predictor for both of the undesirable outcomes $(\mathrm{OR}=2.655 ; 95 \%$ CI $1.121-6.336 ; p=0.027$ and $\mathrm{OR}=6.097 ; 95 \%$ CI 1.756-21.175; $p=0.004$, respectively). Thus, early detection of sarcopenia in patients with IBD is important to prevent undesirable outcomes.
\end{abstract}

Keywords Inflammatory bowel disease $\cdot$ Sarcopenia $\cdot$ Body composition $\cdot$ Surgery $\cdot$ Postoperative complications

\begin{tabular}{|c|c|c|c|}
\hline & & \multicolumn{2}{|c|}{ Abbreviations } \\
\hline & & ASMI & Appendicular skeletal muscle index \\
\hline & & BMI & Body mass index \\
\hline \multirow{3}{*}{\multicolumn{2}{|c|}{$\begin{array}{l}\text { Electronic supplementary material The online version of this } \\
\text { article (https://doi.org/10.1007/s00595-019-01893-8) contains } \\
\text { supplementary material, which is available to authorized users. }\end{array}$}} & $\mathrm{CD}$ & Crohn's disease \\
\hline & & CI & Confidence interval \\
\hline & & CRP & C-reactive protein \\
\hline \multirow{3}{*}{$\Delta$} & \multirow{3}{*}{$\begin{array}{l}\text { Patricia Sarlós } \\
\text { sarlos.patricia@pte.hu }\end{array}$} & CT & Computed tomography \\
\hline & & $\mathrm{ECCO}$ & European Crohn's and colitis organisation \\
\hline & & ESR & Erythrocyte sedimentation rate \\
\hline 1 & $\begin{array}{l}\text { Institute for Translational Medicine, Medical School, } \\
\text { University of Pécs, } 12 \text { Szigeti Street, Pécs 7624, Hungary }\end{array}$ & EWGSOP & $\begin{array}{l}\text { European Working Group on sarcopenia in } \\
\text { older people }\end{array}$ \\
\hline \multirow[t]{2}{*}{2} & Szentágothai Research Centre, University of Pécs, 20 Ifjúság & $\mathrm{Hb}$ & Hemoglobin \\
\hline & Street, Pécs 7624, Hungary & IBD & Inflammatory bowel disease \\
\hline \multirow[t]{2}{*}{3} & Clinical Medicine Doctoral School, University of Szeged, 6 & OR & Odds ratio \\
\hline & Korányi fasor, Szeged 6720, Hungary & SMI & Skeletal muscle index \\
\hline \multirow[t]{3}{*}{4} & Hungarian Academy of Sciences, University of Szeged & TPA & Total psoas muscle area \\
\hline & Momentum Gastroenterology Multidisciplinary Research & $\mathrm{UC}$ & Ulcerative colitis \\
\hline & Group, 8-10 Korányi fasor, Szeged 6720, Hungary & WMD & Weighted mean difference \\
\hline
\end{tabular}

5 First Department of Medicine, Medical School, University of Pécs, 13 Ifjúság Street, Pécs 7624, Hungary

6 First Department of Surgery, Semmelweis University, 78 Üllői Street, Budapest 1082, Hungary

7 Institute of Surgical Research, University of Szeged, 1 Pulz Street, Szeged 6724, Hungary 


\section{Introduction}

The term 'sarcopenia' was introduced by Rosenberg in 1997 to describe an age-related decrease in skeletal muscle mass [1]. According to the recent recommendation of the European Working Group on Sarcopenia in Older People (EWGSOP), sarcopenia can be diagnosed if low lean muscle mass stands together with either low muscle strength or low physical performance [2]. In clinical practice, sarcopenia often remains unrecognized if only body mass index (BMI) is used to determine nutritional status [3]. Several studies have shown that BMI does not predict low lean muscle mass accurately and the entity of sarcopenic obesity also exists [3-5].

Sarcopenia can be assessed anatomically and functionally. Anatomically, a variety of methods and measures are used to evaluate muscle mass, including computed tomography (CT), magnetic resonance imaging (MRI), bioelectrical impedance analysis (BIA), dual-energy X-ray absorptiometry (DXA) and skeletal muscle index (SMI), appendicular SMI (ASMI), and total psoas muscle area (TPA) [2]. However, there is a considerable variance in the cut-off thresholds for sarcopenia in these diagnostic modalities, while the characteristics of the (normal) reference populations also vary [6]. Functionally, the handgrip strength test with a standard dynamometer or a physical performance test such as that using the Short Physical Performance Battery are the gold standards [2]. Sarcopenia in the elderly is often related to adverse health outcomes including a higher risk of hospitalization and mortality with associated increased healthcare costs [7, 8]. Recently, sarcopenia was implicated as a prognostic factor in a wide range of diseases, such as cancer $[9,10]$, chronic liver diseases [11], chronic pancreatitis [12], rheumatic diseases [13] and inflammatory bowel disease (IBD) [14].

Crohn's disease (CD) and ulcerative colitis (UC) are the two main forms of IBD, and both are characterized by chronic inflammation of the digestive tract. Bryant et al. reported low lean muscle mass and sarcopenia in $21 \%$ and $12 \%$ of adult IBD patients, respectively [3]. In a recent systematic review, the incidence of sarcopenia was as high as $52 \%$ in $\mathrm{CD}$ and $37 \%$ in $\mathrm{UC}$, when anatomical criteria were considered without functional strength assessment [14]. Undesired consequences of the altered body composition in IBD include bone demineralization (osteopenia and osteoporosis), inadequate response to therapy, and poor quality of life $[3,15]$. Despite the associations supporting the adverse effect of sarcopenia in many diseases other than IBD, there have been only a few low-volume trials addressing this problem. Thus, we performed this meta-analysis to summarize and synthesize the results of the most up-to-date literature investigating the effect of sarcopenia, as a prognostic factor, on the need for diseaserelated surgery and on the characteristics of postoperative complications in patients with IBD.

\section{Methods}

This meta-analysis was reported in accordance with the Preferred Reporting Items for Systematic Reviews and MetaAnalyses (PRISMA) Statement (Supplementary Table 1) [16]. The protocol was registered in the International Prospective Register of Systematic Reviews (PROSPERO) a priori under registration number CRD42018118517.

\section{Data sources and search strategy}

Our search was conducted in four electronic databases using PubMed (http://www.ncbi.nlm.nih.gov/pubmed), EMBASE (https://www.embase.com), Central Cochrane Register of Controlled Trials (CENTRAL) (http://www.cochraneli brary.com) and Web of Science (www.webofknowledge. com), last updated 13 March 2019. 'English-language' and 'human' filters were applied to the search. A manual search was also done by browsing the reference lists of relevant papers and review articles to identify additional studies. The PECO items of our prognostic meta-analysis were as follows: (P) adult patients with IBD, with available body composition assessment results; (E) those who had diagnosed sarcopenia; and (C) those who did not have sarcopenia. Our outcomes $(\mathrm{O})$ included the number of surgical interventions and postoperative complications. The standardized ClavienDindo classification tool was applied to categorize minor and major postoperative complications [17]. Major complications were defined as grade $\geq$ III on the Clavien-Dindo scale. Studies were identified by entering ('body composition' OR sarcopenia) AND (inflammatory bowel disease or Crohn or ' ulcerative colitis') combining Medical Subject Headings (MeSH) and free-text terms.

\section{Study selection}

After importing all references into a reference management software (EndNote X8, Clarivate Analytics, Philadelphia, PA, US), duplicates and database overlaps were removed by one of the authors (AE). To maximize the precision of selection, the remaining records were screened based on title and abstract, independently, by two of the authors (AE and PS). Finally, the same two authors verified whether the remaining full-text articles or abstracts truly fit the inclusion criteria. If there was disagreement at any stage of the selection, the opinion of a third author (PH) was sought to reach a consensus. English-language full-text articles and conference 
proceedings were eligible for inclusion if they met our inclusion criteria. Uncontrolled studies were excluded.

\section{Data extraction and quality assessment}

From the included studies, two authors extracted the data according to a predefined data abstraction form: first author, year and form of publication (full-text article/abstract only), study design, sample size, and outcome (rate of patients requiring IBD-related surgery and postoperative complications). The definition of sarcopenia and the type of surgery were also recorded (Table 1). Adjusted results generated from, and covariates imputed in, multivariate logistic regression models were also collected (Supplementary Table 2).

We also collected data on patient characteristics such as age, sex, disease duration, BMI, smoking, prior use of immunomodulatory or biological therapies, and preoperatively measured laboratory parameters including hemoglobin $(\mathrm{Hb})$, serum albumin level, C-reactive protein (CRP) and erythrocyte sedimentation rate (ESR) (Table 2). Two independent investigators (AE and PS) performed the quality assessment separately and any disagreements were resolved by discussion. A critical appraisal tool for prognostic studies, the Quality in Prognosis Studies (QUIPS), was used to assess the methodological quality of the studies included [18]. QUIPS covers six main domains, namely, study participation, study attrition, prognostic factor and outcome measurement, study confounding and statistical analysis, and reporting. For each item of the six domains, we used 'yes', 'no', or 'unclear' to assess the risk of bias. Each domain was then judged as carrying 'low', 'moderate' or 'high' risk of bias (Supplementary Table 3).

\section{Statistical analysis}

The Comprehensive MetaAnalysis software Version 3 (Biostat, Inc., Englewood, NJ, USA) was applied to perform meta-analytical calculations with the random effects model [19]. We computed relative measures [odds ratios (ORs) and weighted mean differences (WMDs)] and event rates with $95 \%$ confidence intervals (CIs). The main outcomes, namely, surgical interventions and postoperative complications, were handled as binary variables. First, ORs from raw $2 \times 2$ contingency tables were pooled [19]. Peto's OR was calculated in the case of the study of Carvalho et al. due to rare events [20]. In addition, covariate-adjusted ORs computed with multivariable logistic regression models in the individual studies were pooled [19]. For numerical variables, namely, age, disease duration, BMI, and laboratory parameters, WMDs were calculated. For differences regarding sex, smoking, prior immunomodulator and biologics use between groups, event rates were calculated.
The results of our statistical analysis are shown in tables and forest plots. All analyses were two-tailed and $p<0.05$ was considered significant. For assessing heterogeneity, Cochrane's $Q$ and the $I^{2}$ statistics were used. In the case of the $Q$ statistic, $Q$ exceeds the upper-tail critical value of Chi-square with the $k-1$ degree of freedom. $I^{2}$ represents the percentage of effect size heterogeneity, which cannot be explained by random chance. Based on the cochrane handbook for systematic reviews of interventions, heterogeneity was interpreted as moderate if it was between 30 and $60 \%$, as substantial if it was between 50 and $90 \%$, and as considerable if it was above 75\% [20]. Publication bias was assessed by the visual inspection of the funnel plot, which was complemented with the Egger's test for analysis of the need for surgical interventions [20].

\section{Results}

\section{Search results}

A total of 1260 records were identified from the databases with our systematic search (PubMed: 283; EMBASE: 418; CENTRAL: 39 and Web of Science: 520 articles) (PRISMA flowchart; Fig. 1). Other two potentially eligible articles were identified by our manual search [21, 22]. After removing all duplicates, 735 records remained, 709 of which were excluded based on title and abstract screening. According to our selection criteria, 26 potentially eligible full-text articles and abstracts were considered for final inclusion, 16 of which were excluded, because they (1) failed to report the outcomes of interest $(n=11)$, (2) were systematic reviews $(n=3)$, or (3) were published only in abstract form not containing the required data $(n=2)$. Ten studies were included in the final quantitative analysis [21-30].

\section{Characteristics of the studies included}

Table 1 summarizes the characteristics of the included studies. The trials were published between 2015 and 2018 and all studies were published in full-text form, except for one released as an abstract [28]. The majority were case-control studies and only two prospective cohort studies of Zhang et al. were eligible for meta-analysis [29, 30]. In four studies, patients with $\mathrm{CD}$ were recruited $[21,25,28,30]$, whereas in another three studies, only patients with UC were recruited [26, 27, 29]. In three trials, a mixed cohort of patients with $\mathrm{CD}$ and UC were recruited [22-24].

To quantify the degree of sarcopenia, the majority of studies measured the SMI at the level of the third lumbar vertebra on CT scan, performed within 6 months prior to surgery or in the first postoperative month. The cut-off values of the SMI defining sarcopenia ranged between 39 and 
Table 1 Study characteristics and measured outcomes

\begin{tabular}{|c|c|c|c|c|c|c|c|c|}
\hline \multirow{2}{*}{$\begin{array}{l}\text { Author (year) } \\
\text { study type }\end{array}$} & \multirow{2}{*}{$\begin{array}{l}\text { Number of } \\
\text { patients (CD/ } \\
\text { UC) }\end{array}$} & \multirow{2}{*}{$\begin{array}{l}\text { Definition of sarco- } \\
\text { penia }\end{array}$} & \multirow{2}{*}{$\begin{array}{l}\text { Type of } \\
\text { surgery }\end{array}$} & \multirow{2}{*}{$\begin{array}{l}\text { Number of } \\
\text { patients in } \\
\text { the study } \\
\text { groups }(n)\end{array}$} & \multicolumn{4}{|c|}{ Outcomes (number of patients) } \\
\hline & & & & & Surgery & $\begin{array}{l}\text { Postopera- } \\
\text { tive compli- } \\
\text { cation }\end{array}$ & $\begin{array}{l}\text { Minor } \\
\text { postoperative } \\
\text { complication }\end{array}$ & $\begin{array}{l}\text { Major } \\
\text { postoperative } \\
\text { complication }\end{array}$ \\
\hline \multirow[t]{2}{*}{$\begin{array}{l}\text { Adams [23] } \\
\text { retrosp. }\end{array}$} & \multirow[t]{2}{*}{$90(76 / 14)$} & $\begin{array}{l}\text { Men: } \\
\qquad \mathrm{SMI}<52.4 \mathrm{~cm}^{2} / \mathrm{m}^{2}\end{array}$ & \multirow{2}{*}{$\begin{array}{l}\text { Previous } \\
\text { surgery, not } \\
\text { specified }\end{array}$} & $\begin{array}{l}\text { Sarcopenic } \\
(41)\end{array}$ & 21 & NA & NA & NA \\
\hline & & $\begin{array}{l}\text { Women: } \\
\qquad \mathrm{SMI}<38.5 \mathrm{~cm}^{2} / \mathrm{m}^{2}\end{array}$ & & $\begin{array}{l}\text { Non-sarco- } \\
\text { penic (49) }\end{array}$ & 19 & NA & NA & NA \\
\hline \multirow[t]{2}{*}{$\begin{array}{l}\text { Bamba [24] } \\
\text { retrosp. }\end{array}$} & \multirow[t]{2}{*}{$72(43 / 29)$} & $\begin{array}{l}\text { Men: } \mathrm{SMI}<42 \mathrm{~cm}^{2} / \\
\mathrm{m}^{2}\end{array}$ & \multirow{2}{*}{$\begin{array}{l}\text { Intestinal } \\
\text { resection, } \\
\text { not speci- } \\
\text { fied }\end{array}$} & $\begin{array}{l}\text { Sarcopenic } \\
\text { (30) }\end{array}$ & 16 & NA & NA & NA \\
\hline & & $\begin{array}{l}\text { Women: } \\
\qquad \mathrm{SMI}<38 \mathrm{~cm}^{2} / \mathrm{m}^{2}\end{array}$ & & $\begin{array}{l}\text { Non-sarco- } \\
\text { penic (42) }\end{array}$ & 9 & NA & NA & NA \\
\hline \multirow[t]{2}{*}{$\begin{array}{l}\text { Carvalho [25] } \\
\text { retrosp. }\end{array}$} & \multirow[t]{2}{*}{$58(58 / 0)$} & \multirow{2}{*}{$\begin{array}{l}\text { Men: SMI }<52.4 \\
\mathrm{~cm} 2 / \mathrm{m} 2 \text { Women: } \\
\mathrm{SMI}<38.5 \mathrm{~cm}^{2} / \mathrm{m}^{2}\end{array}$} & \multirow{2}{*}{$\begin{array}{l}\text { Surgical } \\
\text { resection, } \\
\text { not speci- } \\
\text { fied }\end{array}$} & $\begin{array}{l}\text { Sarcopenic } \\
\text { (24) }\end{array}$ & 7 & 7 & NA & NA \\
\hline & & & & $\begin{array}{l}\text { Non-sarco- } \\
\text { penic (34) }\end{array}$ & 17 & 2 & & \\
\hline \multirow[t]{2}{*}{$\begin{array}{l}\text { Cushing [26] } \\
\text { retrosp. }\end{array}$} & \multirow[t]{2}{*}{$82(0 / 82)$} & $\begin{array}{l}\text { Men: } \mathrm{SMI}<55 \mathrm{~cm}^{2} / \\
\mathrm{m}^{2}\end{array}$ & \multirow[t]{2}{*}{ Colectomy } & $\begin{array}{l}\text { Sarcopenic } \\
\text { (57) }\end{array}$ & 16 & NA & NA & NA \\
\hline & & $\begin{array}{l}\text { Women: } \\
\text { SMI }<39 \mathrm{~cm}^{2} / \mathrm{m}^{2}\end{array}$ & & $\begin{array}{l}\text { Non-sarco- } \\
\text { penic }(25)\end{array}$ & 3 & NA & NA & NA \\
\hline \multirow[t]{2}{*}{$\begin{array}{l}\text { Fujikawa [27] } \\
\text { retrosp. }\end{array}$} & \multirow[t]{2}{*}{$69(0 / 69)$} & \multirow{2}{*}{$\begin{array}{l}\text { Men: } \\
\text { TPA }<567.4 \mathrm{~mm}^{2} / \\
\mathrm{m}^{2} \\
\text { Women: } \\
\text { TPA }<355.8 \mathrm{~mm}^{2} / \\
\mathrm{m}^{2}\end{array}$} & \multirow{2}{*}{$\begin{array}{l}\text { Two- or } \\
\text { three- } \\
\text { stage ileal } \\
\text { J-pouch- } \\
\text { anal anas- } \\
\text { tomosis } \\
\text { (IPPA) }\end{array}$} & $\begin{array}{l}\text { Sarcopenic } \\
\text { (18) }\end{array}$ & NA & 8 & 4 & 4 \\
\hline & & & & $\begin{array}{l}\text { Non-sarco- } \\
\text { penic (51) }\end{array}$ & NA & 5 & 3 & 2 \\
\hline \multirow[t]{2}{*}{$\begin{array}{l}\text { O'Brien [22] } \\
\text { retrosp }\end{array}$} & \multirow[t]{2}{*}{$77(52 / 21)^{\mathrm{b}}$} & \multirow{2}{*}{$\begin{array}{l}\text { In case of } \\
\text { BMI }<25 \mathrm{~kg} / \mathrm{m}^{2}: \\
\text { men: } \mathrm{SMI}<43 \mathrm{~cm}^{2} / \\
\mathrm{m}^{2} \\
\text { women: } \\
\mathrm{SMI}<41 \mathrm{~cm}^{2} / \mathrm{m}^{2} \text {; } \\
\text { In case of } \\
\text { BMI } \geq 25 \mathrm{~kg} / \mathrm{m}^{2} \text { : } \\
\text { men: } \mathrm{SMI}<53 \mathrm{~cm}^{2} / \\
\mathrm{m}^{2}\end{array}$} & \multirow[b]{2}{*}{$\begin{array}{l}\text { IPPA, } \\
\text { ileocecec- } \\
\text { tomy, hemi- } \\
\text { colectomy, } \\
\text { colectomy, } \\
\text { panprocto- } \\
\text { colectomy } \\
\text { with or } \\
\text { without } \\
\text { ileostomy } \\
\text { formation }\end{array}$} & $\begin{array}{l}\text { Sarcopenic } \\
\text { (30) }\end{array}$ & 77 & 6 & NA & 6 \\
\hline & & & & $\begin{array}{l}\text { Non-sarco- } \\
\text { penic (47) }\end{array}$ & & 10 & & 10 \\
\hline \multirow[t]{2}{*}{$\begin{array}{l}\text { Oh [28] } \\
\text { retrosp. }\end{array}$} & \multirow[t]{2}{*}{$79(79 / 0)$} & $\begin{array}{l}\text { Men: } \mathrm{SMI}<55 \mathrm{~cm}^{2} / \\
\mathrm{m}^{2}\end{array}$ & \multirow[t]{2}{*}{$\begin{array}{l}\text { Surgery, not } \\
\text { specified }\end{array}$} & $\begin{array}{l}\text { Sarcopenic } \\
\text { (64) }\end{array}$ & 11 & NA & NA & NA \\
\hline & & $\begin{array}{l}\text { Women: } \\
\qquad \mathrm{SMI}<39 \mathrm{~cm}^{2} / \mathrm{m}^{2}\end{array}$ & & $\begin{array}{l}\text { Non-sarco- } \\
\text { penic (15) }\end{array}$ & 2 & NA & NA & NA \\
\hline \multirow[t]{2}{*}{$\begin{array}{l}\text { Thiberge [21] } \\
\text { retrosp. }\end{array}$} & \multirow[t]{2}{*}{$149(149 / 0)$} & $\begin{array}{l}\text { Men: } \\
\text { SMI }<55.4 \mathrm{~cm}^{2} / \mathrm{m}^{2}\end{array}$ & \multirow[b]{2}{*}{$\begin{array}{l}\text { Stric- } \\
\text { turoplasty, } \\
\text { bowel } \\
\text { resection, } \\
\text { stoma } \\
\text { without } \\
\text { resection, } \\
\text { perianal/ } \\
\text { abdominal } \\
\text { abscess } \\
\text { drainage, } \\
\text { viscerolysis }\end{array}$} & $\begin{array}{l}\text { Sarcopenic } \\
\quad(50)\end{array}$ & $\begin{array}{l}\text { OR: } 2.03^{\mathrm{a}} \text {, } \\
\quad(\mathrm{CI}\end{array}$ & NA & NA & NA \\
\hline & & $\begin{array}{l}\text { Men: } \\
\qquad \mathrm{SMI}<38.9 \mathrm{~cm}^{2} / \mathrm{m}^{2}\end{array}$ & & $\begin{array}{l}\text { Non-sarco- } \\
\text { penic (99) }\end{array}$ & $\begin{array}{l}0.98-4.26) \\
p=0.056\end{array}$ & NA & NA & NA \\
\hline
\end{tabular}


Table 1 (continued)

\begin{tabular}{|c|c|c|c|c|c|c|c|c|}
\hline \multirow{2}{*}{$\begin{array}{l}\text { Author (year) } \\
\text { study type }\end{array}$} & \multirow{2}{*}{$\begin{array}{l}\text { Number of } \\
\text { patients (CD/ } \\
\text { UC) }\end{array}$} & \multirow{2}{*}{$\begin{array}{l}\text { Definition of sarco- } \\
\text { penia }\end{array}$} & \multirow{2}{*}{$\begin{array}{l}\text { Type of } \\
\text { surgery }\end{array}$} & \multirow{2}{*}{$\begin{array}{l}\text { Number of } \\
\text { patients in } \\
\text { the study } \\
\text { groups }(n)\end{array}$} & \multicolumn{4}{|c|}{ Outcomes (number of patients) } \\
\hline & & & & & Surgery & $\begin{array}{l}\text { Postopera- } \\
\text { tive compli- } \\
\text { cation }\end{array}$ & $\begin{array}{l}\text { Minor } \\
\text { postoperative } \\
\text { complication }\end{array}$ & $\begin{array}{l}\text { Major } \\
\text { postoperative } \\
\text { complication }\end{array}$ \\
\hline \multirow[t]{2}{*}{$\begin{array}{l}\text { Zhang [29] } \\
\text { prosp. }\end{array}$} & \multirow[t]{2}{*}{$114(114 / 0)$} & $\begin{array}{l}\text { Men: } \mathrm{SMI}<55 \mathrm{~cm}^{2} / \\
\mathrm{m}^{2}\end{array}$ & \multirow{2}{*}{$\begin{array}{l}\text { Segmen- } \\
\text { tal/total } \\
\text { colectomy, } \\
\text { ileocecal/ } \\
\text { small bowel } \\
\text { resection }\end{array}$} & $\begin{array}{l}\text { Sarcopenic } \\
\text { (70) }\end{array}$ & NA & 32 & 21 & 11 \\
\hline & & $\begin{array}{l}\text { Women: } \\
\qquad \mathrm{SMI}<39 \mathrm{~cm}^{2} / \mathrm{m}^{2}\end{array}$ & & $\begin{array}{l}\text { Non-sarco- } \\
\text { penic (44) }\end{array}$ & NA & 26 & 25 & 1 \\
\hline \multirow[t]{2}{*}{$\begin{array}{l}\text { Zhang [30] } \\
\text { prosp. }\end{array}$} & \multirow[t]{2}{*}{$99(0 / 99)$} & $\begin{array}{l}\text { Men: } \mathrm{SMI}<55 \mathrm{~cm}^{2} / \\
\mathrm{m}^{2}\end{array}$ & \multirow[t]{2}{*}{ Colectomy } & $\begin{array}{l}\text { Sarcopenic } \\
\text { (27) }\end{array}$ & 7 & NA & NA & NA \\
\hline & & $\begin{array}{l}\text { Women: } \\
\qquad \mathrm{SMI}<39 \mathrm{~cm}^{2} / \mathrm{m}^{2}\end{array}$ & & $\begin{array}{l}\text { Non-sarco- } \\
\text { penic (72) }\end{array}$ & 7 & NA & NA & NA \\
\hline
\end{tabular}

$C D$ crohn's disease, $U C$ ulcerative colitis, $S M I$ skeletal muscle index, TPA total psoas muscle area, $O R$ odds ratio, $C I$ confidence interval, $p p$ value, retrosp retrospective, prosp prospective, $N A$ non-available

${ }^{a}$ No raw data available

${ }^{\mathrm{b}}$ Additional four cases of indeterminate colitis

$41 \mathrm{~cm}^{2} / \mathrm{m}^{2}$ for women and between 52 and $55 \mathrm{~cm}^{2} / \mathrm{m}^{2}$ for men. Bamba et al. used standardized SMI cut-off values for liver disease with a lower SMI for men $\left(42 \mathrm{~cm}^{2} / \mathrm{m}^{2}\right)$ [24]. Only one study applied TPA for sarcopenia assessment [27].

Whilst seven studies compared IBD patients with sarcopenia versus those without sarcopenia in relation to need for surgery [21, 23-26, 28, 29], only four studies published results on complications occurring in the first month after surgery [22, 25, 27, 30]. A total of 275 IBD-related operations were performed in the studies included. A high diversity of surgical interventions was registered, including stricturoplasty, bowel resection, abscess drainage, viscerolysis, ileal J-pouch-anal anastomosis, and various types of colectomy.

\section{Patients' characteristics}

Altogether, 885 patients with IBD were included in this meta-analysis: 571 (64.5\%) with CD and 314 (35.5\%) with UC. Based on the body composition measurements, $46.2 \%$ (409/885) of the patients had sarcopenia. There were significantly more men in the sarcopenic group than in the nonsarcopenic group $(66.8 \%$ vs. $46.3 \%, p=0.027$; Tables 2 and 3). Patients with IBD and sarcopenia had significantly lower BMI and preoperative serum albumin levels and significantly higher CRP levels than the non-sarcopenic patients (WMD: $2.698 \mathrm{~kg} / \mathrm{m}^{2}, 95 \%$ CI $1.507-3.889, p<0.001 ; I^{2}=52.39 \%$, $p=0.078$; WMD: $3.276 \mathrm{~g} / \mathrm{dL}, 95 \%$ CI $0.022-0.623$, $p=0.035 ; I^{2}=90.74 \%, p<0.001$ and WMD: $12.740 \mathrm{mg} / \mathrm{L}$, $95 \%$ CI 7.154-18.326, $p<0.001 ; I^{2}=0 \%, p=0.524$, respectively). There were no significant differences in age, disease duration, smoking habits, or prior immunomodulator or biologics use between the sarcopenic and the non-sarcopenic groups (Table 3).

Analyzing preoperative laboratory studies (serum albumin and CRP) and other patient characteristics (such as the rate of sarcopenia, BMI, SMI, and height) by IBD subtypes revealed that the rate of sarcopenia was significantly higher in patients with CD than in those with UC $(60.7 \%$ vs. $36.7 \%, p=0.044$ ) [24, 29]. The preoperative serum albumin level was also significantly higher in those with $\mathrm{CD}$ than in those with UC (WMD: $0.337 \mathrm{~g} / \mathrm{dL}, 95 \%$ CI 0.055-0.619, $\left.p=0.019 ; I^{2}=67.53 \%, p=0.079\right)$ (Supplementary Table 4$)$ [24, 29].

\section{Need for surgery}

Seven studies assessed the need for surgical intervention in IBD patients with versus those without sarcopenia [21, 23-26, 28, 29]. Overall, 35.5\% (104/293) of the sarcopenic and $27.9 \%$ (94/336) of the non-sarcopenic patients underwent disease-related surgery, respectively. The raw data on the number of sarcopenic and non-sarcopenic patients with IBD who underwent surgery were available in six studies [23-26, 28, 29]. Analysis of the unadjusted data showed no significant difference between the two groups with respect to the need for surgical interventions (unadjusted OR: 1.826; 95\% CI 0.913-3.654; $p=0.089)$. Moderate heterogeneity was detected across the studies $\left(I^{2}=54.62 \%, p=0.051\right)$ (Fig. 2). Three studies were adjusted for significant covariates [21, 26, 29]. After pooling the adjusted ORs together, sarcopenia proved to be an independent predictor of the need for surgery (adjusted OR: 2.665; 95\% CI 1.121-6.336; $p=0.027$ ), with moderate between-study heterogeneity $\left(I^{2}=33.94 \%, p=0.220\right)$ (Fig. 3). 


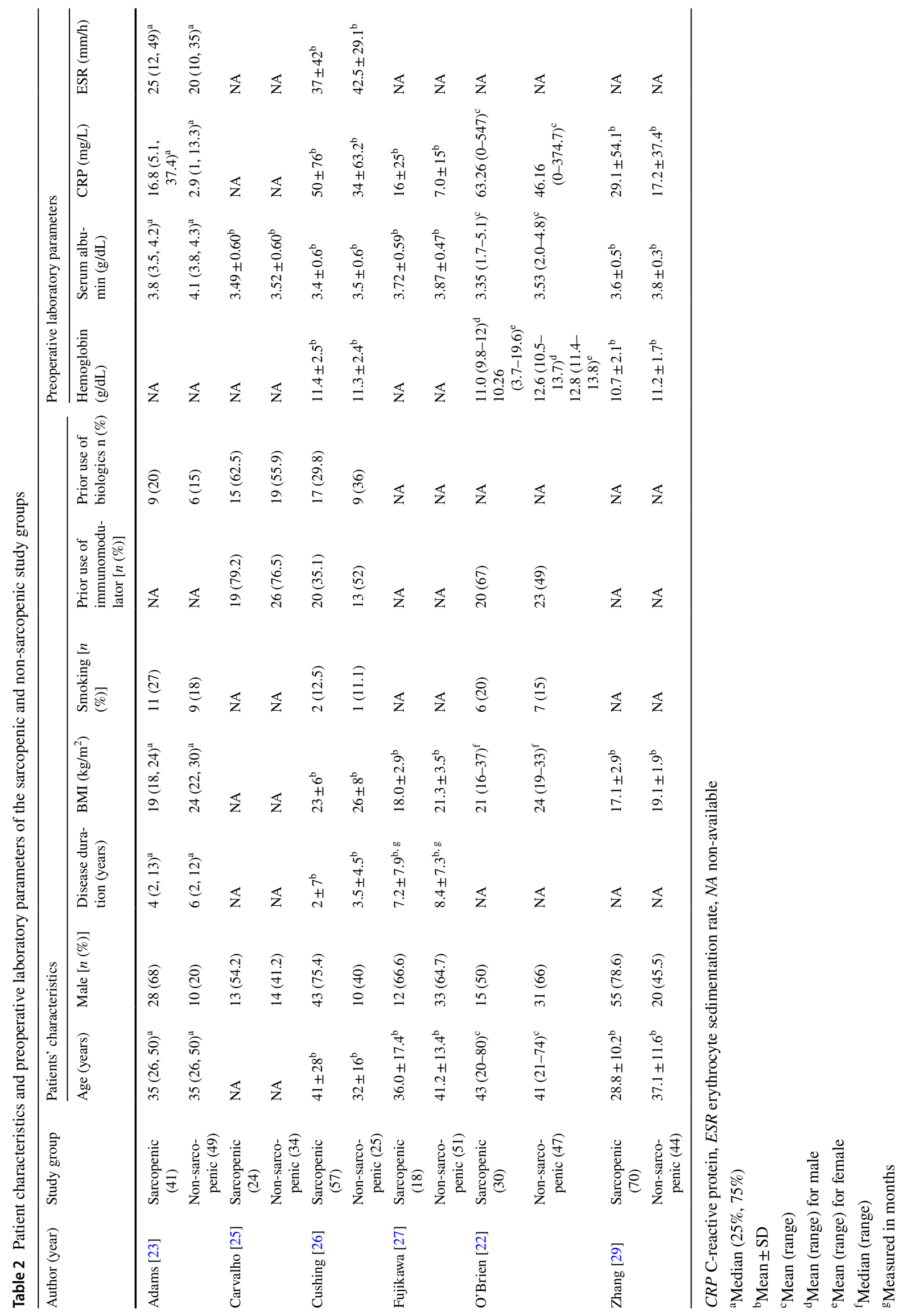


Fig. 1 Flow diagram of the study selection process

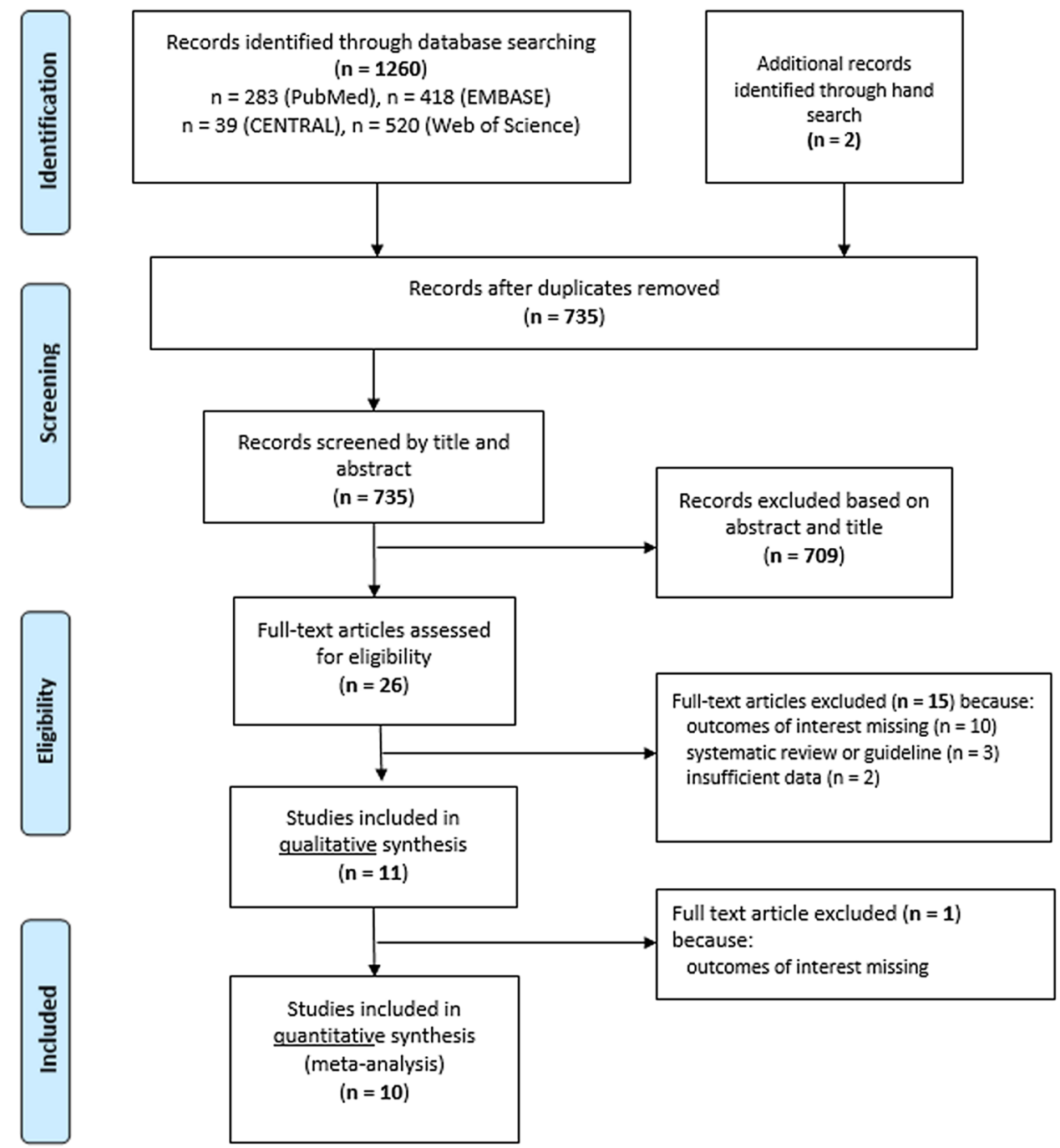

When the data of the CD and UC patients were analyzed separately, no significant difference was found between the sarcopenic and non-sarcopenic groups in the rate of surgical intervention (unadjusted OR: 1.566; 95\% CI 0.415-5.904; $p=0.508 ; I^{2}=81.65 \%, p=0.004$ and unadjusted OR: 2.931 ; 95\% CI 0.760-11.309; $p=0.119 ; I^{2}=0.0 \%, p=0.978$, respectively) (Fig. 4).

\section{Postoperative complications}

Four studies were eligible for analysis of postoperative complications in IBD patients with versus those without sarcopenia [22, 25, 27, 30] (Fig. 5a). Postoperative complications developed in $37.3 \%(53 / 142)$ of the sarcopenic patients versus $24.4 \%$ (43/176) of the non-sarcopenic group, without a significant difference between the two groups (unadjusted OR: 3.265; 95\% CI 0.575-18.557; $p=0.182$; $\left.I^{2}=88.46 \%, p<0.001\right)$. Two studies were adjusted for significant covariates $[27,30]$. After pooling the adjusted ORs together, sarcopenia proved to be an independent predictor of postoperative complications (adjusted OR $=6.097 ; 95 \%$ CI 1.756-21.175; $p=0.004)$, with negligible between-study heterogeneity $\left(I^{2}=0.0 \%, p=0.637\right)$ (Fig. 6).

Performing subgroup analysis based on the ClavienDindo grading system, sarcopenia was not a risk factor of minor or major postoperative complications (unadjusted OR: 1.107; 95\% CI: 0.084-14.632; $p=0.939$, and unadjusted OR: 3.124 ; 95\% CI: 0.666-14.652; $p=0.149$, respectively) (Fig. 5b, c). Considerable heterogeneity was observed in the analyses of overall, minor, and major postoperative complications $\left(I^{2}=88.46 \%, p<0.001 ; I^{2}=88.01 \%, p=0.004\right.$; and $I^{2}=62.31 \%, p=0.070$, respectively).

\section{Risk of bias assessment}

Supplementary Figs. 1 and 2 summarize the results of the risk of bias assessment of the individual studies and Supplementary Fig. 3 shows the adapted QUIPS tool. The 
Table 3 Heterogeneity of data on the differences in patient characteristics and laboratory parameters of the sarcopenic and non-sarcopenic study groups

\begin{tabular}{|c|c|c|c|c|c|c|c|c|}
\hline & \multirow{2}{*}{$\begin{array}{l}\text { Difference } \\
\text { in means }\end{array}$} & \multicolumn{2}{|l|}{$95 \% \mathrm{CI}$} & \multirow[t]{2}{*}{$p$ value } & \multicolumn{4}{|c|}{ Heterogeneity } \\
\hline & & Lower limit & Upper limit & & $Q$ value & $d f(Q)$ & $p$ value & $I^{2}$ \\
\hline Age & -1.432 & -7.215 & 4.351 & 0.627 & 14.260 & 4 & 0.007 & 71.95 \\
\hline Disease duration (months) & -1.441 & -5.403 & 2.522 & 0.476 & 0.861 & 2 & 0.650 & 0.0 \\
\hline BMI & -2.698 & -3.889 & -1.507 & $<0.001$ & 8.401 & 4 & 0.078 & 52.39 \\
\hline Preoperative $\mathrm{Hb}$ & -3.437 & -9.502 & 2.951 & 0.302 & 0.730 & 1 & 0.393 & 0.0 \\
\hline Preoperative serum albumin & -3.276 & -0.623 & -0.022 & $\mathbf{0 . 0 3 5}$ & 53.97 & 5 & 0.000 & 90.74 \\
\hline Preoperative CRP & 12.740 & 7.154 & 18.326 & $<0.001$ & 3.204 & 4 & 0.524 & 0.0 \\
\hline \multirow[t]{3}{*}{ Preoperative ESR } & 3.163 & -8.137 & 14.463 & 0.583 & 1.407 & 1 & 0.236 & 28.94 \\
\hline & Event rate & \multicolumn{2}{|l|}{$95 \% \mathrm{CI}$} & \multirow[t]{2}{*}{$p$ value } & \multicolumn{4}{|c|}{ Heterogeneity } \\
\hline & & Lower limit & Upper limit & & $Q$ value & $d f(Q)$ & $p$ value & $I^{2}$ \\
\hline Sex, male: sarcopenic & 0.668 & 0.541 & 0.775 & 0.027 & 11.247 & 5 & 0.047 & 55.44 \\
\hline Sex, male: non-sarcopenic & 0.463 & 0.338 & 0.593 & & 25.723 & 5 & 0.000 & 80.56 \\
\hline Smoking: sarcopenic & 0.146 & 0.081 & 0.247 & 0.786 & 8.326 & 2 & 0.016 & 75.99 \\
\hline Smoking: non-sarcopenic & 0.137 & 0.056 & 0.286 & & 2.426 & 2 & 0.297 & 17.55 \\
\hline Prior immunomodulators: sarcopenic & 0.593 & 0.411 & 0.752 & 0.400 & 7.544 & 2 & 0.023 & 73.45 \\
\hline Prior immunomodulators: non-sarcopenic & 0.484 & 0.316 & 0.657 & & 6.403 & 2 & 0.041 & 68.77 \\
\hline Prior biologics: sarcopenic & 0.354 & 0.260 & 0.461 & 0.815 & 10.769 & 1 & 0.005 & 81.43 \\
\hline Prior biologics: non-sarcopenic & 0.341 & 0.258 & 0.434 & & 15.742 & 2 & 0.000 & 87.23 \\
\hline
\end{tabular}

$B M I$ body mass index, $H g$ hemoglobin, $C R P$ C-reactive protein, $E S R$ erythrocyte sedimentation rate, $C I$ confidence interval, $d f$ degree of freedom

Associations significant at $p<0.05$ vs controls shown in bold

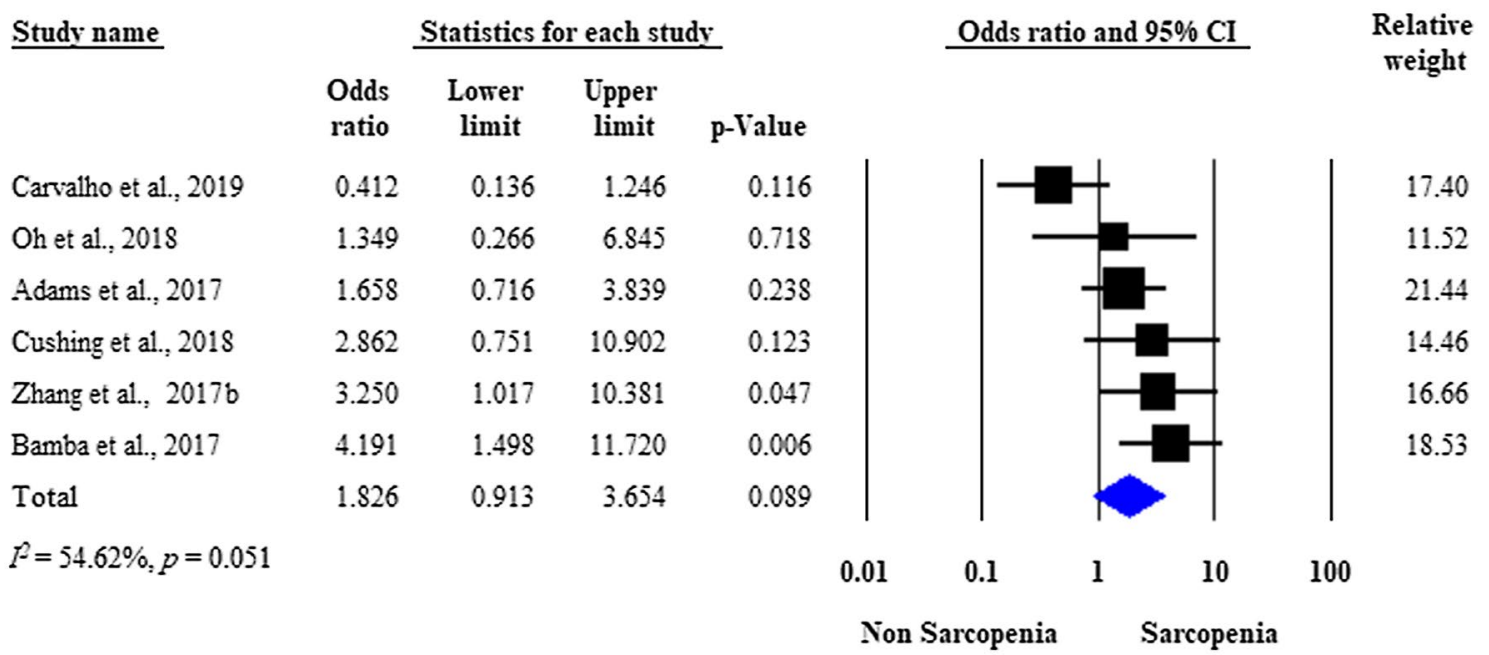

Fig. 2 Forest plot of studies evaluating inflammatory bowel disease (IBD)-related surgical interventions in the sarcopenic and non-sarcopenic study groups (unadjusted results). Size of squares for the odds ratio reflects the weight of the trial in pooled analyses. Horizontal bars represent $95 \%$ confidence intervals

two domains classified as carrying high and one domain classified as carrying a moderate risk of bias. All the studies included were assessed as carrying a high risk of bias in at least one domain. The domain of 'prognostic factor measurement' was the best rated, as all the included studies

of bias, while the study of Oh showed the worst results with 


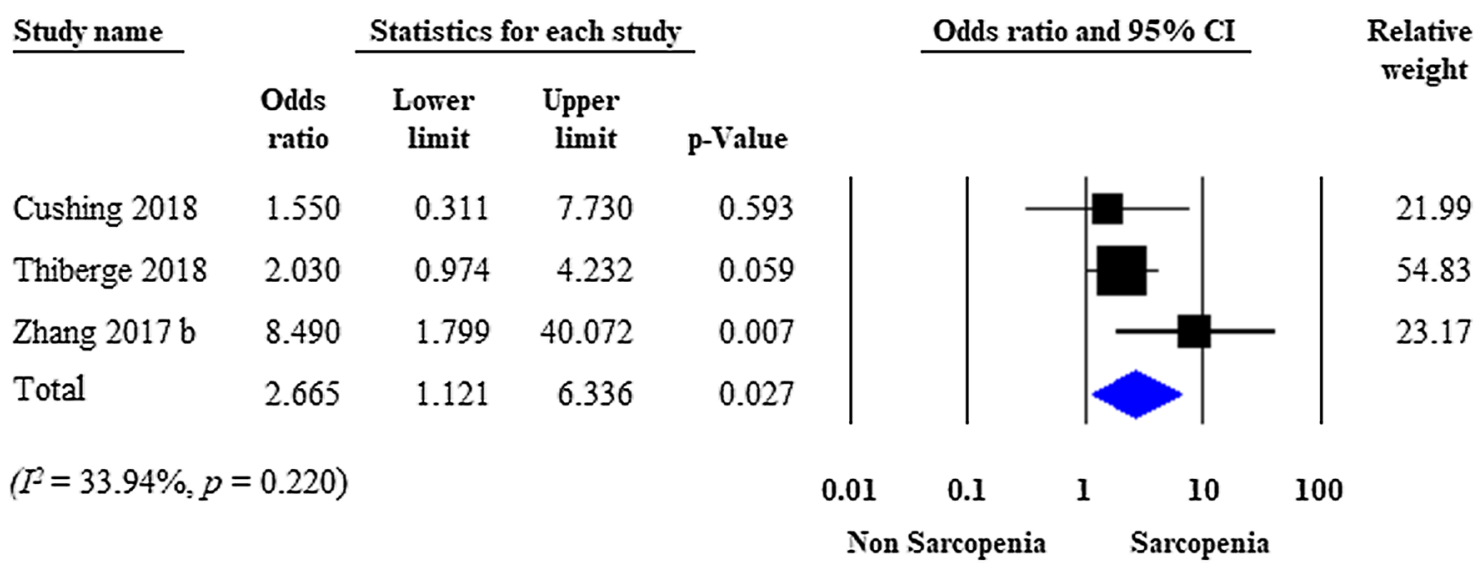

Fig. 3 Forest plot of studies evaluating IBD-related surgical interventions in the sarcopenic and non-sarcopenic study groups (adjusted results). Size of squares for the odds ratio reflects the weight of the trial in pooled analyses. Horizontal bars represent $95 \%$ confidence intervals

\begin{tabular}{|c|c|c|c|c|c|}
\hline \multirow[t]{2}{*}{ Study name } & \multirow[t]{2}{*}{ Subgroup within study } & \multicolumn{4}{|c|}{ Statistics for each study } \\
\hline & & $\begin{array}{l}\text { Odds } \\
\text { ratio }\end{array}$ & $\begin{array}{l}\text { Lorrer } \\
\text { limit }\end{array}$ & $\begin{array}{c}\text { Upper } \\
\text { limit }\end{array}$ & $\mathrm{p}-\mathrm{Yal}_{\mathrm{l}}$ \\
\hline Bamba et a!. 2017 & $\mathrm{UC}$ & 2.600 & 0.394 & 17.159 & \\
\hline Cushing et al. 2018 & $\mathrm{UC}$ & 2.862 & 0.751 & 10.902 & \\
\hline Znang et a1. 2016 & $\mathrm{UC}$ & 3.250 & 1.017 & 10.381 & \\
\hline \multicolumn{2}{|c|}{ UC Total $\left(\mathrm{I}^{2}=0 \% ; p=0.978\right)$} & 2931 & 0.760 & 11.309 & \\
\hline Carvalho et al. 2019 & CD & 0.412 & 0.136 & 1.246 & \\
\hline Onet a1. 2018 & CD & 1.349 & 0.266 & 6.845 & \\
\hline Bamba et a1. 2017 & $\mathrm{CD}$ & 8.571 & 2.068 & 35.523 & \\
\hline \multicolumn{2}{|c|}{ CD Total $\left(\mathrm{I}^{2}=81.65 \% ; p=0.004\right)$} & 1.566 & 0.415 & 5.904 & \\
\hline \multicolumn{2}{|c|}{ Overall $\left(\mathrm{I}^{2}=61.79 \% ; p=0.023\right)$} & 2.131 & 0.827 & 5.490 & \\
\hline
\end{tabular}

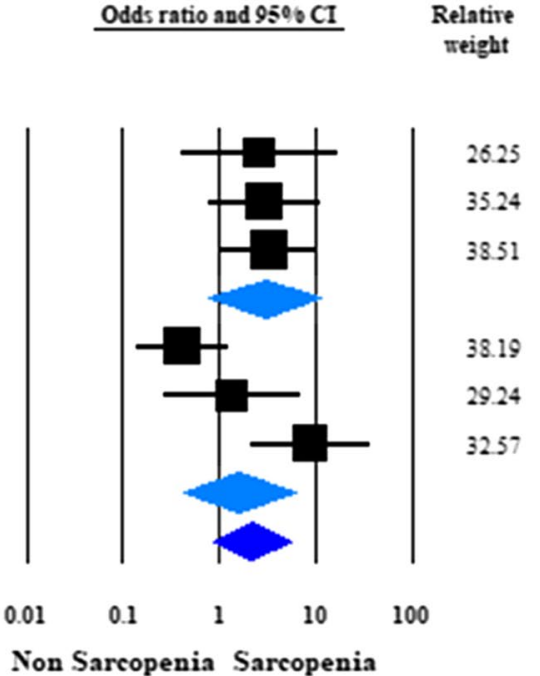

Fig. 4 Forest plots of studies evaluating the effect of sarcopenia on disease-related surgical interventions in the Crohn's disease (CD) and ulcerative colitis (UC) patient populations (unadjusted results). Size

were judged to carry a low risk of bias. In contrast, $90 \%$ of the studies showed a high risk of bias in the term of 'study confounding', since they did not report how the important confounders were adjusted for and whether an appropriate method was used for handling missing data. In all the studies evaluated, 'study participation' and 'outcome measurement' domains carried low or moderate risk of bias. Most of the studies (90\%) detailed statistical analysis properly and were, therefore, awarded a green notation in this regard.

\section{Publication bias}

Based on visual assessment of the funnel plot (symmetric) and the Egger's test result $(p=0.91)$, a 'small-study effect' of squares for odds ratio reflects weight of trial in pooled analysis. Horizontal bars represent $95 \%$ confidence intervals. UC ulcerative colitis, $C D$ Crohn's disease

is unlikely to occur in the analysis of the need for surgical intervention (Supplementary Fig. 3).

\section{Discussion}

Sarcopenia is relevant for patients with IBD as it can lead to poor outcomes, such as bone demineralization with consequential pathological fractures, hospitalization, reduced mobility, and compromised quality of life [31]. Apart from the increased level of pro-inflammatory mediators, inadequate calorie intake, malabsorption, and protein-losing enteropathy, different pharmacological and surgical treatments may also impair nutritional status in IBD [15]. 
Study name

(a) All complications

Zhang et a1., $2017 \mathrm{a}$
OBrien et al., 2018
Fujikawa et a1., 2015
Carvalho et a1., $2019^{*}$
Total
$\left(I^{2}=\$ 8.46 \%, p<0.001\right)$

(b) Minor complications

Zhang et al., 2017a
Fujikawa et al., 2015
Total
$\left(I^{2}=\$ 8.01 \%, p=0.004\right)$

(c) Major complications

$\begin{array}{llll}\text { O'Brien et al., 2018 } & 0.925 & 0.297 & 2.878 \\ \text { Fujikawa et a1., 2015 } & 7.000 & 1.159 & 42.276 \\ \text { Zhang et a1., 2017a } & 8.017 & 0.997 & 64.461 \\ \text { Total } & 3.124 & 0.666 & 14.652 \\ \left(I^{2}=62.31 \% p=0.070\right) & & & \end{array}$

*: Peto's odds ratio was calculated due to rare events

Fig. 5 Forest plot of studies evaluating postoperative complications in the sarcopenic and non-sarcopenic patients with IBD (unadjusted results). a All complications; $\mathbf{b}$ minor complications were defined as grade I-II; c major complications were defined as grade $\geq$ III on the odds ratio and $95 \%$ CI Relative

weight

p-Value
27.44

25.82

24.55

22.19

0.182

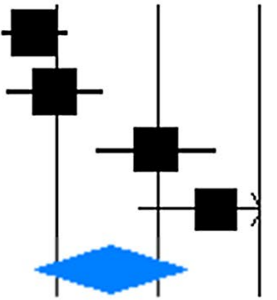

3.69

46.31

100.00

0.939
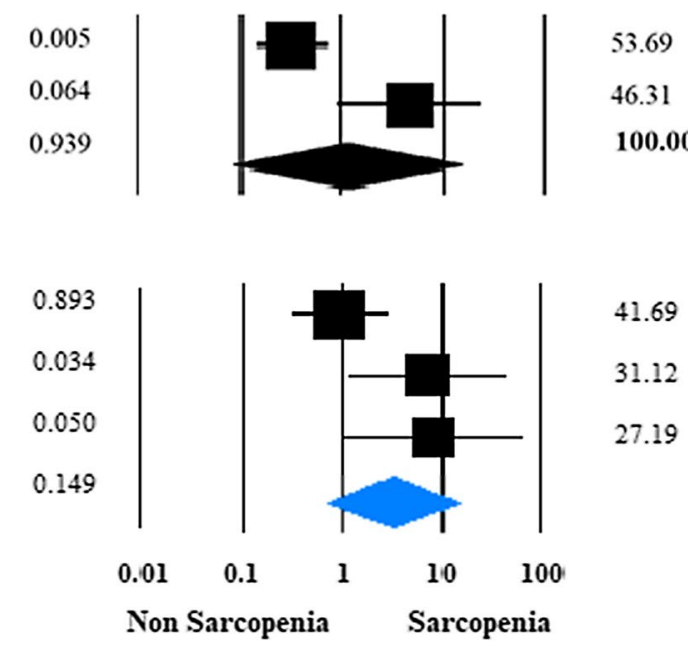

Clavien-Dindo scale. Size of squares for the odds ratio reflects the weight of the trial in pooled analyses. Horizontal bars represent $95 \%$ confidence intervals

\begin{tabular}{lccc} 
Study name & & Statistics for each st \\
\cline { 3 - 5 } & $\begin{array}{c}\text { Odds } \\
\text { ratio }\end{array}$ & $\begin{array}{c}\text { Lower } \\
\text { limit }\end{array}$ & $\begin{array}{c}\text { Upper } \\
\text { limit }\end{array}$ \\
Fujikawa et al.; 2015 & 4.910 & 1.057 & 22.798 \\
Zhang et al.; 2017a & 9.240 & 1.101 & 77.558 \\
Total & 6.097 & 1.756 & 21.175 \\
$\left(\mathrm{I}^{2}=0.0 \% ; p=0.637\right)$ & & &
\end{tabular}

Fig. 6 Forest plot of studies evaluating postoperative complications in the sarcopenic and non-sarcopenic patients with IBD considering all complications (adjusted results). Size of squares for the odds ratio

\section{Odds ratio and $95 \% \mathrm{CI}$ \\ Relative \\ weight}

p-Value

0.042
0.041
0.004

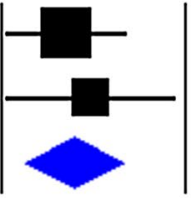

65.75

34.25

\section{$\begin{array}{lllll}0.01 & 0.1 & 1 & 10 & 100\end{array}$}

Non Sarcopenia Sarcopenia

reflects the weight of the trial in pooled analyses. Horizontal bars represent $95 \%$ confidence intervals 
However, few studies have evaluated the effect of sarcopenia as a prognostic factor of surgical outcomes.

Our meta-analysis found only seven studies with controversial results on surgical interventions for sarcopenic patients with IBD. Although we did not detect a significant difference between the rate of surgical interventions for the sarcopenic patients versus the non-sarcopenic patients with IBD when unadjusted data were pooled, our analysis of covariate-adjusted data identified sarcopenia as an independent predictor of surgical interventions in patients with IBD. A potential explanation for this phenomenon could be the effect of bias (especially selection bias), which masked the true difference in the unadjusted analysis (Figs. 2 and 5), but was reduced, at least partly, with the introduction of multivariate models (Figs. 3 and 6). Regarding IBD subtype (CD or UC), no significant difference was observed in surgical interventions, although there was a trend towards a more frequent need for surgery in sarcopenic patients with UC.

Similarly, we could only detect significant differences between the groups with respect to postoperative complications when adjusted data were analyzed. This finding is consistent with previous meta-analyses on patients who underwent oncological abdominal surgery, where radiologically proven sarcopenia was associated with a significant increase in major postoperative complications as well as in 30-day mortality [32, 33]. In our meta-analysis, most of the sarcopenic patients were men. Interestingly, a previous study identified sarcopenia as a predictor of a subsequent surgery only in women with IBD [34]. Other clinical features such as age, disease duration, smoking, and prior therapies did not differ considerably between the groups. We also found lower BMI and serum albumin levels and higher CRP levels in the sarcopenic group. It should be noted that measuring these parameters can be helpful in the prediction of sarcopenia. Since cross-sectional imaging is frequently ordered preoperatively, the results of CT or MRI scans would provide a more accurate estimation of lean muscle mass.

Although the consequences of malnutrition, such as poor bone health, delayed puberty and growth failure, are more prevalent in pediatric patients with IBD, our meta-analysis highlighted that the assessment of body composition is also necessary in adult patients with IBD [35]. The importance of evaluating the nutritional status of an IBD patient was emphasized in the recent European Crohn's and Colitis Organisation (ECCO) guidelines [36, 37]. Malnutrition and hypalbuminaemia are listed as the main risk factors of postoperative complications, including anastomotic leak, peritonitis, and intra-abdominal septic complications [36]. Prior to surgery, not only the responsible adjustment of medical therapy, such as weaning off steroid treatment if possible, but also preoperative enteral or parenteral nutritional support may help to reduce the risk of surgical and postoperative complications [38-42].
Several limitations of this meta-analysis must be considered. First, the vast majority of included studies were retrospective with a small sample size and a wide variety of surgical interventions, raising concerns about imprecision and indirectness. Second, none of the included studies used EWGSOP criteria to assess functional loss of muscle strength measurement. Third, only one study assessed the effect of nutritional therapy, in which preoperative enteral nutrition was a protective factor against major postoperative complications [30]. Furthermore, minor differences were observed with respect to the covariates imputed in the logistic regression models, which may affect our results (Supplementary Table 2). As heterogeneity tests indicated homogeneous datasets (Figs. 3 and 6), we do not suspect rough distortion. Finally, there was considerable heterogeneity in cut-off points for the sarcopenia definition regarding ethnicity, carrying the potential of under- or overestimating the sarcopenia rate in the included studies.

The main strength of our work is its novelty, but we must mention the foremost systematic review by Ryan et al. [14] investigating the prognostic role of sarcopenia in the outcomes of surgery for IBD. Our review revealed the need for longitudinal observational studies in this field. The highly transparent and reproducible methodology of this work was ensured by strictly adhering to the rules and recommendations of the PRISMA guidelines.

In conclusion, the findings of our analysis have implications for practice, particularly in the promotion of preoperative individualized risk prediction. In addition to simple anthropometric tests, anatomical and functional measurements should be performed. The SMI, measured on a CT scan, can be used as an objective assessment tool to identify sarcopenia in patients with IBD. To interpret the body composition and nutritional status of patients with IBD, a multidisciplinary approach is recommended. Education on nutritional issues is best provided by well-trained dietitians with a special interest in IBD. Since sarcopenia may be reversible with adequate nutritional support, targeted preoperative risk reduction strategies are recommended to optimize surgical outcomes. Further research through large prospective cohort studies is needed to confirm our findings and conclusions.

Acknowledgements Open access funding provided by University of Pécs (PTE)

Author contribution Conceptualization, AE and PS; methodology, EA, PS and AS; formal analysis, AS; investigation, AE and PS; resources, Peter $\mathrm{H}$; writing - original draft preparation, AE and PS; writing review and editing, $\mathrm{AS}, \mathrm{ZS}, \mathrm{MB}, \mathrm{AS}$, Petra $\mathrm{H}, \mathrm{BE}$; visualization, $\mathrm{AE}$, $\mathrm{PS}$ and $\mathrm{ZS}$; supervision, BE and Peter $\mathrm{H}$; project administration, $\mathrm{ZS}$; funding acquisition, Peter $\mathrm{H}$.

Funding This work was supported by an Economic Development and Innovation Operative Programme Grant, GINOP 2.3.2-15-2016-00048 to PH; a Human Resources Development Operational Programme of 
the European Union and the Hungarian Government EFOP-3.6.2-162017-0006 to PH.

\section{Compliance with ethical standards}

Conflicts of interest We have no conflicts of interest to declare.

Open Access This article is distributed under the terms of the Creative Commons Attribution 4.0 International License (http://creativeco mmons.org/licenses/by/4.0/), which permits unrestricted use, distribution, and reproduction in any medium, provided you give appropriate credit to the original author(s) and the source, provide a link to the Creative Commons license, and indicate if changes were made.

\section{References}

1. Rosenberg IH. Sarcopenia: origins and clinical relevance. J Nutr. 1997;127(5 Supp1):990S-1S. https://doi.org/10.1093/ jn/127.5.990S.

2. Cruz-Jentoft AJ, Baeyens JP, Bauer JM, Boirie Y, Cederholm T, Landi F, et al. Sarcopenia: European consensus on definition and diagnosis: report of the European Working Group on sarcopenia in older people. Age Ageing. 2010;39(4):412-23. https://doi. org/10.1093/ageing/afq034.

3. Bryant RV, Ooi S, Schultz CG, Goess C, Grafton R, Hughes J, et al. Low muscle mass and sarcopenia: common and predictive of osteopenia in inflammatory bowel disease. Aliment Pharmacol Ther. 2015;41(9):895-906. https://doi.org/10.1111/apt.13156.

4. Bryant RV, Trott MJ, Bartholomeusz FD, Andrews JM. Systematic review: body composition in adults with inflammatory bowel disease. Aliment Pharmacol Ther. 2013;38(3):213-25. https://doi. org/10.1111/apt.12372.

5. Bryant RV, Schultz CG, Ooi S, Goess C, Costello SP, Vincent AD, et al. Obesity in inflammatory bowel disease: gains in adiposity despite high prevalence of myopenia and osteopenia. Nutrients. 2018. https://doi.org/10.3390/nu10091192.

6. Bahat G, Tufan A, Tufan F, Kilic C, Akpinar TS, Kose M, et al. Cut-off points to identify sarcopenia according to European Working Group on Sarcopenia in Older People (EWGSOP) definition. Clin Nutr. 2016;35(6):1557-63. https://doi.org/10.1016/j. clnu.2016.02.002.

7. Beaudart C, Zaaria M, Pasleau F, Reginster JY, Bruyere O. Health outcomes of sarcopenia: a systematic review and meta-analysis. PLoS One. 2017;12(1):e0169548. https://doi.org/10.1371/journ al.pone. 0169548 .

8. Steffl M, Sima J, Shiells K, Holmerova I. The increase in health care costs associated with muscle weakness in older people without long-term illnesses in the Czech Republic: results from the Survey of Health, Ageing and Retirement in Europe (SHARE). Clin Interv Aging. 2017;12:2003-7. https://doi.org/10.2147/CIA. S150826.

9. Fukushima H, Takemura K, Suzuki H, Koga F. Impact of sarcopenia as a prognostic biomarker of bladder cancer. Int J Mol Sci. 2018. https://doi.org/10.3390/ijms19102999.

10. Lee JS, Kim YS, Kim EY, Jin W. Prognostic significance of CTdetermined sarcopenia in patients with advanced gastric cancer. PLoS One. 2018;13(8):e0202700. https://doi.org/10.1371/journ al.pone. 0202700 .

11. Hsu CS, Kao JH. Sarcopenia and chronic liver diseases. Expert Rev Gastroenterol Hepatol. 2018;12(12):1229-44. https://doi. org/10.1080/17474124.2018.1534586.
12. Olesen SS, Buyukuslu A, Kohler M, Rasmussen HH, Drewes AM. Sarcopenia associates with increased hospitalization rates and reduced survival in patients with chronic pancreatitis. Pancreatology. 2019;19(2):245-51. https://doi.org/10.1016/j. pan.2019.01.006.

13. Barone M, Viggiani MT, Anelli MG, Fanizzi R, Lorusso O, Lopalco G, et al. Sarcopenia in patients with rheumatic diseases: prevalence and associated risk factors. J Clin Med. 2018. https:// doi.org/10.3390/jcm7120504.

14. Ryan E, McNicholas D, Creavin B, Kelly ME, Walsh T, Beddy D. Sarcopenia and inflammatory bowel disease: a systematic review. Inflamm Bowel Dis. 2019;25(1):67-73. https://doi.org/10.1093/ ibd/izy212.

15. Scaldaferri F, Pizzoferrato M, Lopetuso LR, Musca T, Ingravalle F, Sicignano LL, et al. Nutrition and IBD: malnutrition and/or sarcopenia? A practical guide. Gastroenterol Res Pract. 2017;2017:8646495. https://doi.org/10.1155/2017/8646495.

16. Moher D, Liberati A, Tetzlaff J, Altman DG, Group P. Preferred reporting items for systematic reviews and meta-analyses: the PRISMA statement. Int J Surg. 2010;8(5):336-41. https://doi. org/10.1016/j.ijsu.2010.02.007.

17. Dindo D, Demartines N, Clavien PA. Classification of surgical complications: a new proposal with evaluation in a cohort of 6336 patients and results of a survey. Ann Surg. 2004;240(2):205-13.

18. Hayden JA, van der Windt DA, Cartwright JL, Cote P, Bombardier C. Assessing bias in studies of prognostic factors. Ann Intern Med. 2013;158(4):280-6. https://doi.org/10.7326/0003-4819-1584-201302190-00009.

19. DerSimonian R, Laird N. Meta-analysis in clinical trials. Control Clin Trials. 1986;7(3):177-88.

20. Higgins JP, Altman DG, Gotzsche PC, Juni P, Moher D, Oxman $\mathrm{AD}$, et al. The cochrane collaboration's tool for assessing risk of bias in randomised trials. BMJ. 2011;343:d5928. https://doi. org/10.1136/bmj.d5928.

21. Thiberge C, Charpentier C, Gillibert A, Modzelewski R, Dacher JN, Savoye G, et al. Lower subcutaneous or visceral adiposity assessed by abdominal computed tomography could predict adverse outcome in patients with Crohn's disease. J Crohns Colitis. 2018;12(12):1429-37. https://doi.org/10.1093/ecco-jcc/jjy12 4

22. O'Brien S, Kavanagh RG, Carey BW, Maher MM, O'Connor OJ, Andrews EJ. The impact of sarcopenia and myosteatosis on postoperative outcomes in patients with inflammatory bowel disease. Eur Radiol Exp. 2018;2(1):37. https://doi.org/10.1186/s4174 7-018-0072-3.

23. Adams DW, Gurwara S, Silver HJ, Horst SN, Beaulieu DB, Schwartz DA, et al. Sarcopenia is common in overweight patients with inflammatory bowel disease and may predict need for surgery. Inflamm Bowel Dis. 2017;23(7):1182-6. https://doi. org/10.1097/MIB.0000000000001128.

24. Bamba S, Sasaki M, Takaoka A, Takahashi K, Imaeda H, Nishida A, et al. Sarcopenia is a predictive factor for intestinal resection in admitted patients with Crohn's disease. PLoS One. 2017;12(6):e0180036. https://doi.org/10.1371/journal.pone.01800 36.

25. Carvalho D, Viana C, Marques I, Costa C, Martins SF. Sarcopenia is associated with postoperative outcome in patients with Crohn's disease undergoing bowel resection. Gastrointest Disord. 2019;1(1):201-9.

26. Cushing KC, Kordbacheh H, Gee MS, Kambadakone A, Ananthakrishnan AN. Sarcopenia is a novel predictor of the need for rescue therapy in hospitalized ulcerative colitis patients. J Crohns Colitis. 2018. https://doi.org/10.1093/ecco-jcc/jjy064.

27. Fujikawa H, Araki T, Okita Y, Kondo S, Kawamura M, Hiro $\mathrm{J}$, et al. Impact of sarcopenia on surgical site infection after 
restorative proctocolectomy for ulcerative colitis. Surg Today. 2017;47(1):92-8. https://doi.org/10.1007/s00595-016-1357-x.

28. Oh DJ, Yoon H, Choi YJ, Shin CM, Park YS, Kim N, et al. The prevalence of sarcopenia and its effect on prognosis in patients with Crohn's disease. J Crohns Colitis. 2018;12:S222.

29. Zhang T, Ding C, Xie T, Yang J, Dai X, Lv T, et al. Skeletal muscle depletion correlates with disease activity in ulcerative colitis and is reversed after colectomy. Clin Nutr. 2017;36(6):1586-92. https://doi.org/10.1016/j.clnu.2016.10.004.

30. Zhang T, Cao L, Cao T, Yang J, Gong J, Zhu W, et al. Prevalence of sarcopenia and its impact on postoperative outcome in patients with crohn's disease undergoing Bowel resection. JPEN J Parenter Enter Nutr. 2017;41(4):592-600. https://doi.org/10.1177/01486 07115612054.

31. Santilli V, Bernetti A, Mangone M, Paoloni M. Clinical definition of sarcopenia. Clin Cases Min Bone Metab. 2014;11(3):177-80.

32. Jones K, Gordon-Weeks A, Coleman C, Silva M. Radiologically determined sarcopenia predicts morbidity and mortality following abdominal surgery: a systematic review and meta-analysis. World J Surg. 2017;41(9):2266-79. https://doi.org/10.1007/s0026 8-017-3999-2.

33. Simonsen C, de Heer P, Bjerre ED, Suetta C, Hojman P, Pedersen BK, et al. Sarcopenia and postoperative complication risk in gastrointestinal surgical oncology: a meta-analysis. Ann Surg. 2018;268(1):58-69. https://doi.org/10.1097/SLA.0000000000 002679 .

34. Gurwara S, Silver HJ, Calloway AP, Horst SN, Beaulieu D, Schwartz DA, et al. Sarcopenia is common in IBD patients and may predict need for surgery. Gastroenterology. 2016;150(4):S403-4.

35. Gerasimidis K, McGrogan P, Edwards CA. The aetiology and impact of malnutrition in paediatric inflammatory bowel disease. J Hum Nutr Diet. 2011;24(4):313-26. https://doi.org/10.1111/ j.1365-277X.2011.01171.x.

36. Bemelman WA, Warusavitarne J, Sampietro GM, Serclova Z, Zmora O, Luglio G, et al. ECCO-ESCP consensus on surgery for
Crohn's disease. J Crohns Colitis. 2018;12(1):1-16. https://doi. org/10.1093/ecco-jcc/jjx061.

37. Oresland T, Bemelman WA, Sampietro GM, Spinelli A, Windsor A, Ferrante M, et al. European evidence based consensus on surgery for ulcerative colitis. J Crohns Colitis. 2015;9(1):4-25. https://doi.org/10.1016/j.crohns.2014.08.012.

38. Gu J, Stocchi L, Remzi F, Kiran RP. Factors associated with postoperative morbidity, reoperation and readmission rates after laparoscopic total abdominal colectomy for ulcerative colitis. Colorectal Dis. 2013;15(9):1123-9. https://doi.org/10.1111/codi.12267.

39. Markel TA, Lou DC, Pfefferkorn M, Scherer LR 3rd, West K, Rouse T, et al. Steroids and poor nutrition are associated with infectious wound complications in children undergoing first stage procedures for ulcerative colitis. Surgery. 2008;144(4):540-5. https://doi.org/10.1016/j.surg.2008.07.005 (Discussion 545-547).

40. Li Y, Zuo L, Zhu W, Gong J, Zhang W, Gu L, et al. Role of exclusive enteral nutrition in the preoperative optimization of patients with Crohn's disease following immunosuppressive therapy. Medicine (Baltimore). 2015;94(5):e478. https://doi.org/10.1097/ MD.0000000000000478.

41. Schwartz E. Perioperative parenteral nutrition in adults with inflammatory bowel disease: a review of the literature. Nutr Clin Pract. 2016;31(2):159-70. https://doi.org/10.1177/0884533615 594011.

42. Jacobson S. Early postoperative complications in patients with Crohn's disease given and not given preoperative total parenteral nutrition. Scand J Gastroenterol. 2012;47(2):170-7. https://doi. org/10.3109/00365521.2011.648954.

Publisher's Note Springer Nature remains neutral with regard to jurisdictional claims in published maps and institutional affiliations. 